\title{
A novel modeling approach for finite element human body models with high computational efficiency and stability: application in pedestrian safety analysis
}

\author{
GUIBING Li ${ }^{1}$, HeNGSHUAi MENG ${ }^{1}$, JiNMING LIU $^{1}$, DONGHUA ZOU ${ }^{2}, \mathrm{KUI} \mathrm{LI}^{3}$ \\ ${ }^{1}$ School of Mechanical Engineering, Hunan University of Science and Technology, Xiangtan, China. \\ ${ }^{2}$ Shanghai Key Laboratory of Forensic Medicine, Academy of Forensic Science, Ministry of Justice, Shanghai, China. \\ ${ }^{3}$ Chongqing Key Laboratory of Vehicle Crash/Bio-Impact and Traffic Safety, Institute for Traffic Medicine, \\ Daping Hospital, Army Medical University, Chongqing, China.
}

\begin{abstract}
Purpose: The purpose of the current study was to develop and validate a finite element (FE) pedestrian model with high computational efficiency and stability using a novel modeling approach. Methods: Firstly, a novel modeling approach of using hollow structures (HS) to simulate the mechanical properties of soft tissues under impact loading was proposed and evaluated. Then, an FE pedestrian model was developed, employing this modeling approach based on the Total Human Model for Safety (THUMS) pedestrian model, named as THUMS-HS model. Finally, the biofidelity of the THUMS-HS model was validated against cadaver test data at both segment and full-body level. Results: The results show that the proposed hollow structures can simulate the mechanical properties of soft tissues and the predictions of the THUMS-HS model show good agreement with the cadaver test data under impact loading. Simulations also prove that the THUMS-HS model has high computational efficiency and stability. Conclusions: The proposed modeling approach of using hollow structures to simulate the mechanical properties of soft tissues is plausible and the THUMS-HS model could be used as a valid, efficient and robust numerical tool for analysis of pedestrian safety in vehicle collisions.
\end{abstract}

Key words: FE human body modeling, computational efficiency and stability, pedestrian safety

\section{Introduction}

Biomechanical studies of human injury mechanism and prevention have provided useful references for vehicle safety evaluation and design, where finite element (FE) human body models have become one of the most important tools. During the past few decades, many FE human body models have been developed and applied in vehicle safety analysis, including segment and full-scale body models [9], [16], [24], [31], [32]. For full-scale human body models, the GHBMC adult male 50th percentile models [23], [25] and Total Human Model for Safety (THUMS) models in different postures and sizes [21] are popular in vehicle safety analysis. These models are generally well developed with detailed anatomical structures and have been fully validated against cadaver test data [21], [23] [25]. However, there are still some limitations in these detailed human body models for expensive simulations (e.g., large-scale or long duration simulations) and high impact energy scenarios (e.g., high impact speed), given the low computational efficiency (long computing time) and stability (usually error termination due to negative volume of solid elements). Previous studies have focused on improving the computational efficiency of these detailed human body models using simplified geometry and large sized solid elements for modeling the soft tissues, such as subcutaneous fat, skeletal muscle and organs [10], [23]. But

\footnotetext{
* Corresponding author: Guibing Li, School of Mechanical Engineering, Hunan University of Science and Technology, Xiangtan, China. E-mail: 1i8747@hotmail.com

Received: January 22nd, 2021

Accepted for publication: March 16th, 2021
} 
this simplification method could not eliminate error termination from negative volume of solid elements in high energy crash simulations. Furthermore, the high quality requirement for solid elements from solving precision also increases the difficulty of model development [31].

On the other hand, current vehicle evaluation in the New Car Assessment Programs (NCAP) for pedestrian protection only focuses on impact speeds at or below $40 \mathrm{~km} / \mathrm{h}$ [1], [2]. Though accident analysis implies that current vehicles are safer than the past [12], [13], [19], the fatality risk at $40 \mathrm{~km} / \mathrm{h}$ is only about 5\% [18] and more than 270000 pedestrians are still killed on the road every year [29]. Current pedestrian protection regulations should raise their requirements to focus on impact scenarios with a relatively higher fatality risk, such as crashes at an impact speed significantly higher than $40 \mathrm{~km} / \mathrm{h}$. Biomechanical analysis of pedestrian safety in crashes at high impact speeds would be helpful. Unfortunately, this kind of study is scarce and our preliminary attempts on this were failed due to error terminations of simulations for negative volume of solid elements in the pedestrian model. Thus, FE pedestrian models with high computational stability in high impact energy scenarios are on demand.

Therefore, the purpose of the current study was to develop an FE pedestrian model with high computational efficiency and stability. Firstly, a modeling approach of using hollow structures to simulate the mechanical properties of soft tissues under impact loading was proposed. Then, a pedestrian model was developed applying the hollow structure modeling approach to modify the subcutaneous fat and skeletal muscle in the THUMS AM50 Version 5.0 model (mentioned as THUMS model hereinafter), and full validation against cadaver test data was carried out for this modified model.

\section{Materials and methods}

\subsection{Basic concept of using hollow structures in human body modeling}

Hollow structures (or honeycomb structures) are widely used in mechanical devices for their superior strength/weight relationship and good energy absorption properties [4], [14]. In FE modeling, hollow structures can be simulated using shell elements [4], which have higher computational efficiency and stability compared to solid elements under crash loading [15], [31]. Thus, the current work proposes the concept of using hollow structures to simulate the mechanical properties of human body soft tissues. To evaluate this concept, basic impact simulations were carried out using LS-DYNA codes [15], where the solid and hollow structures $($ size $=180 \mathrm{~mm} \times 180 \mathrm{~mm} \times 90 \mathrm{~mm}$ ) were struck by a rigid impactor (diameter $=90 \mathrm{~mm}$, weight $=5 \mathrm{~kg}$ and initial velocity $=40 \mathrm{~km} / \mathrm{h}$ ) (Fig. 1). In the simulations, the bottom surface of the solid and hollow structures was fixed. The solid structure was modeled by solid elements and rubber material (MAT 181 in LS-DYNA) [15], which is commonly used for simulating soft tissues in human body modeling [17], [21]. The material properties used for the skeletal muscle (upper and lower limbs) and subcutaneous fat (thorax and abdomen) in the THUMS model were employed to the solid structure, respectively (Table 1). The hollow structure was modeled as grids in size of $30 \mathrm{~mm}$ $\times 30 \mathrm{~mm} \times 30 \mathrm{~mm}$ using shell elements in thickness of $1.5 \mathrm{~mm}$, and plastic material was defined since this is a computationally efficient model in FE modeling [15]. A optimization was conducted to find the Young's modulus and yield stress (Table 1) for the plastic material of the hollow structures that can predict the mechanical properties of the solid structures of skeletal muscle and subcutaneous fat, respectively. In the optimization framework, direct communication between the genetic algorithm (GA) and simulation input/output was built and the fitness (calculated by the least square method) of the force-displacement curve between a hollow and corresponding solid structure was defined as the optimization objective. The reliability of this optimization framework has been proved in our previous study [19]. The predicted deformation and force-displacement curves were compared between the solid and hollow structures (Fig. 2), where the hollow structures can generally predicted the mechanical properties of soft tissues under impact loading. Thus, this hollow structure modeling approach is plausible.
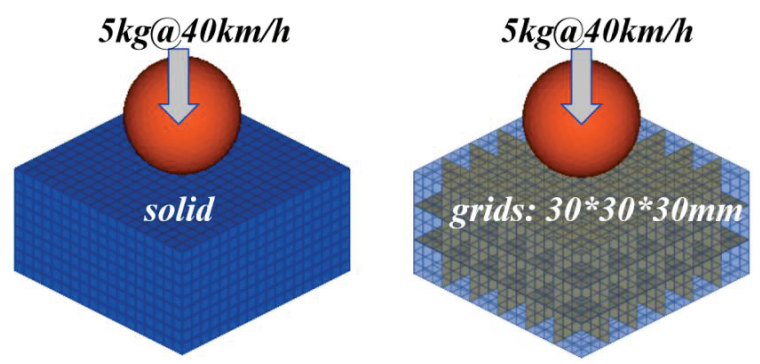

Fig. 1. Basic impact simulations for the solid and hollow structures 
Table 1. Material properties for the solid and hollow structures

\begin{tabular}{|l|l|l|c|c|c|c|}
\hline \multicolumn{1}{|c|}{ Structure } & $\begin{array}{c}\text { Material } \\
\text { model }\end{array}$ & \multicolumn{1}{|c|}{ Body part } & $\begin{array}{c}\text { Linear } \\
\text { bulk modulus }\end{array}$ & $\begin{array}{c}\text { Young's } \\
\text { modulus }\end{array}$ & $\begin{array}{c}\text { Yield } \\
\text { stress }\end{array}$ & $\begin{array}{c}\text { Force-displacement } \\
\text { curve }\end{array}$ \\
\hline \multirow{2}{*}{ Solid } & \multirow{2}{*}{ Rubber } & Skeletal muscle & $1000 \mathrm{MPa}$ & $/$ & $/$ & Fig. A1 \\
\cline { 3 - 7 } & Subcutaneous fat & $4.59 \mathrm{MPa}$ & $/$ & $/$ & Fig. A2 \\
\hline \multirow{2}{*}{ Hollow } & \multirow{2}{*}{ Plastic } & Skeletal muscle & $/$ & $100 \mathrm{MPa}$ & $4 \mathrm{MPa}$ & $/$ \\
\cline { 3 - 7 } & Subcutaneous fat & $/$ & $50 \mathrm{MPa}$ & $2 \mathrm{MPa}$ & $/$ \\
\hline
\end{tabular}

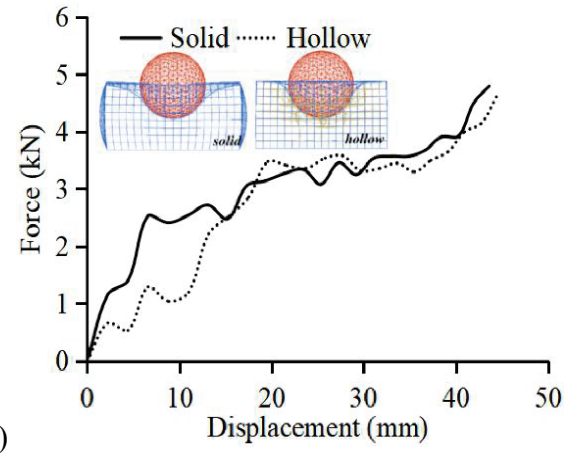

(a)

Fig. 2. Predicted force-displacement curves for the solid and hollow structures: skeletal muscle (a) and subcutaneous fat (b)

\subsection{FE pedestrian model with hollow structures}

Based on the above modeling approach, an FE pedestrian model (named as THUMS-HS model) was developed by replacing the subcutaneous fat and skeletal muscle in the THUMS model with hollow structures (Fig. 3). To improve the computing efficiency further, the bones in upper and lower limbs of the THUMS-HS model were simulated using singlelayer shell elements. The head, neck and bones and organs in thorax and abdomen were kept as solid parts, as defined in the THUMS model. The THUMS-HS model contains about 500,000 shell elements (400 000 in the THUMS) and 760000 solid elements (1 400000 in the THUMS). The material properties for the hollow

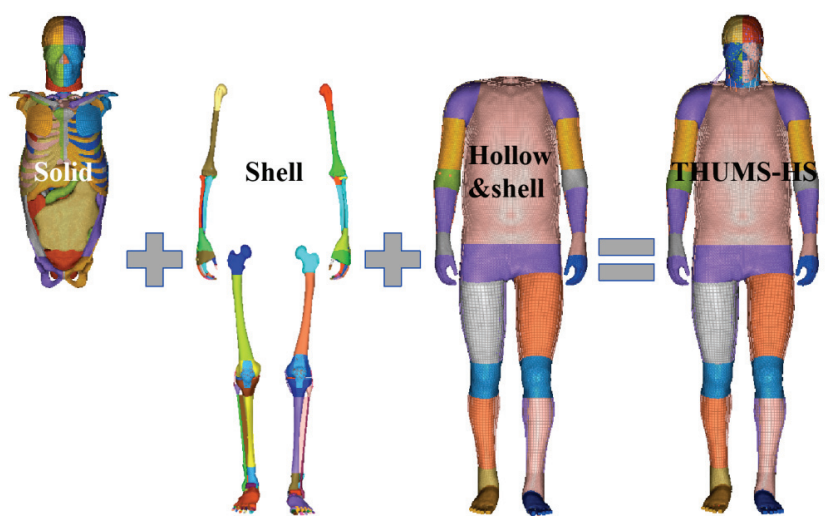

Fig. 3. THUMS model with hollow structures (THUMS-HS) structures listed in Table 1 were defined to the skeletal muscle (upper and lower limbs) and subcutaneous fat (thorax and abdomen) accordingly in the THUMS-HS model. For the bones in upper and lower limbs, elastoplastic material model and material properties reported in a previous study [32] were defined.

\subsection{THUMS-HS model validation}

Validation of the THUMS-HS model focuses on both segment and full body levels, similarly to previous studies [10], [23]. For the leg and thigh, the dynamic three point bending tests from Kerrigan et al. [6] were simulated using the THUMS-HS model, where the loading position was set at the middle of the leg/thigh and the loading direction was at the $\mathrm{L}-\mathrm{M}$ direction with a speed of $1.5 \mathrm{~m} / \mathrm{s}$ (Fig. 4).

Cadaver impact tests to the pelvis, abdomen, thorax and shoulder from previous studies [8], [26] were simulated for validation to the torso (Fig. 5). The impactor was $23.4 \mathrm{~kg}$ in weight and $150 \mathrm{~mm}$ in diameter for tests to the pelvis, abdomen, and thorax. The impactor for the shoulder had the same dimensions but a mass of $23 \mathrm{~kg}$. For each body part, the same impact direction and location as the tests were defined, and two impact velocities used in the cadaver tests were employed. In the pelvis validation, the forearm was removed from the full body model to avoid interference, but the corresponding mass was attached to the elbow to keep the inertial force. 
(a)

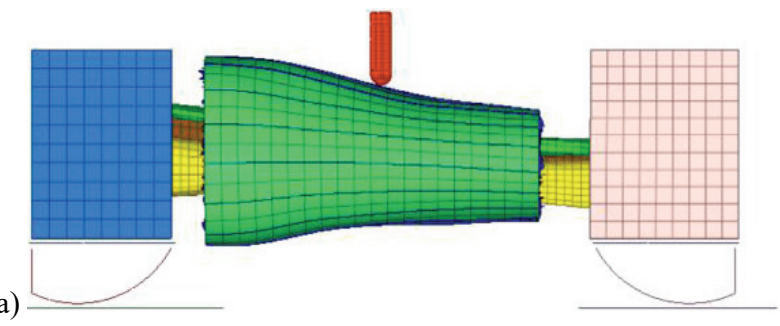

(b)

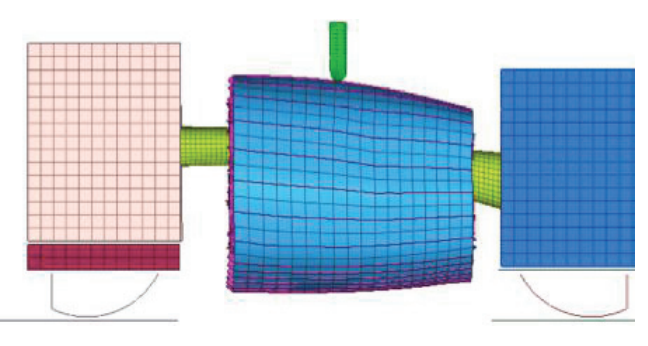

Fig. 4. Leg (a) and thigh (b) dynamic three point bending test setup

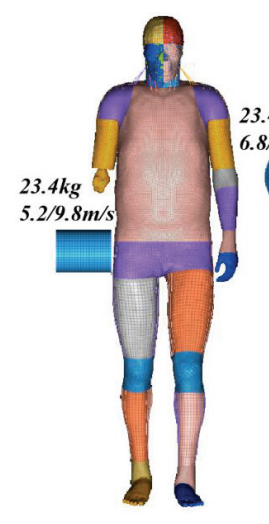

(a)

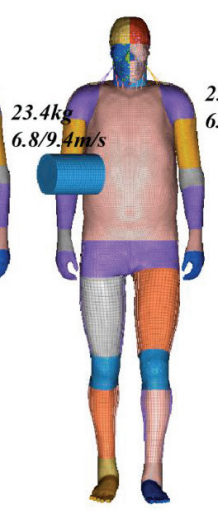

(b)

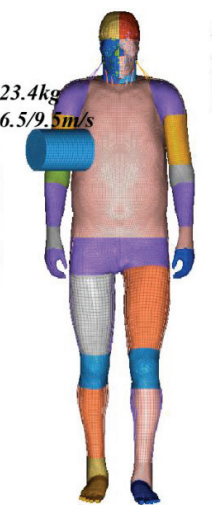

(c)

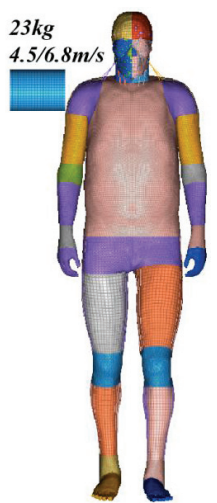

(d)
Fig. 5. Pelvis (a), abdomen (b), thorax (c) and shoulder (d) impact tests setup

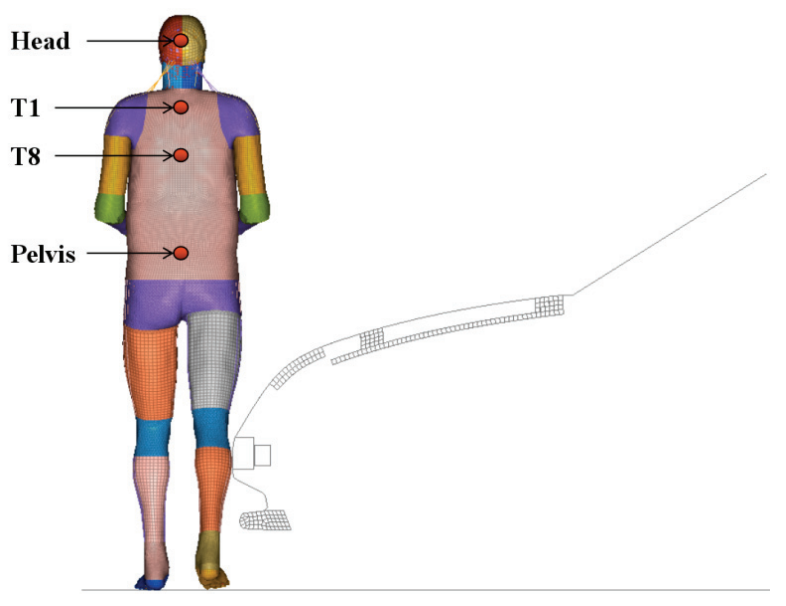

Fig. 6. Car-to-pedestrian impact test setup

For the validation at the full-body level, a vehicle-to-pedestrian impact simulation was defined according to the cadaver test setup in Kerrigan et al. [7] (Fig. 6). In the simulation, a simplified sedan front model in the same geometry as the car used in the cadaver tests was employed, which was the simplification approach for car front models used in previous studies [10], [22], [30]. The hands of the THUMS-HS model were tied in front and the legs were set in a walking posture. The impact velocity was at $40 \mathrm{~km} / \mathrm{h}$ from the right sight of the model. The trajectories of head, T1, T8 and pelvis were extracted from the simulation according to the corresponding locations of the record mark fixation points in the tests [7]. The correlation between the predictions and cadaver test data was assessed quantitatively using the CORA (correlation analysis) method, similarly to previous studies [10], [23], [33]. In the CORA, the corridor method (CORA-CD) calculates the deviation between the predicting curve and the reference corridors, while the cross-correlation method (CORA-CL) evaluates specific curve fitness to the target through parameters [3]. A CORA rating closer to $100 \%$ indicates a better fitness.

\section{Results}

\subsection{Validation at the segment level}

In Figure 7, the predicted force-displacement and moment-displacement curves from the leg and thigh of the THUMS-HS model together with the corridors from cadaver tests were shown. For both leg and thigh, the predictions from the THUMS-HS model were within the corridors of cadaver test data (adapted from Ivarsson et al. [5]).
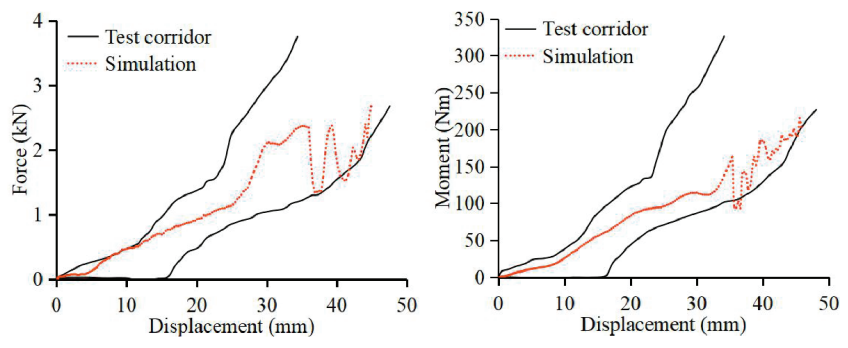

(a)
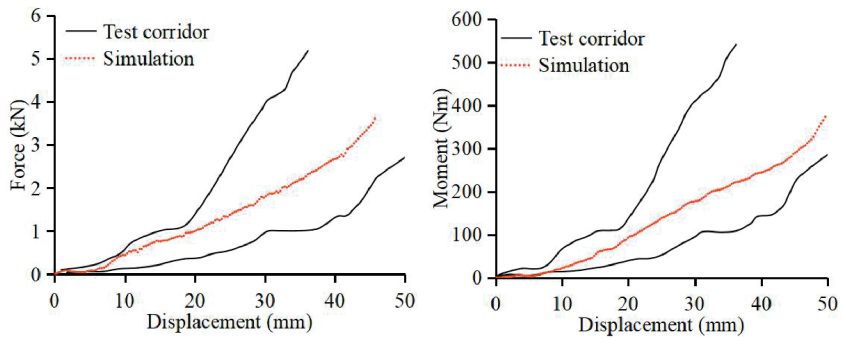

Fig. 7. Force-displacement and moment-displacement curves predicted from THUMS-HS versus cadaver data: (a) leg and (b) thigh 

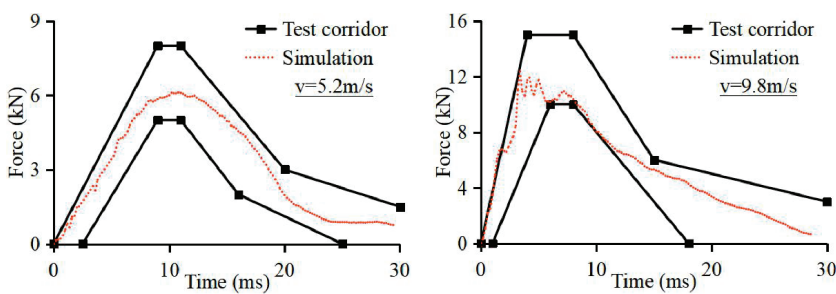

(a)
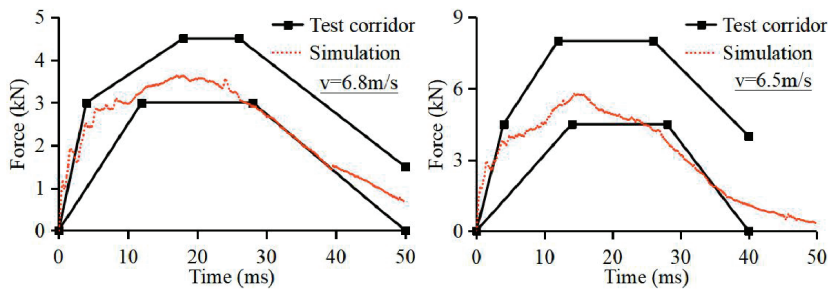

(b)
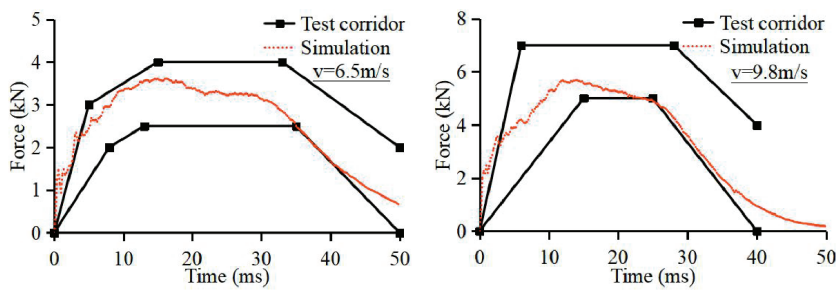

(c)
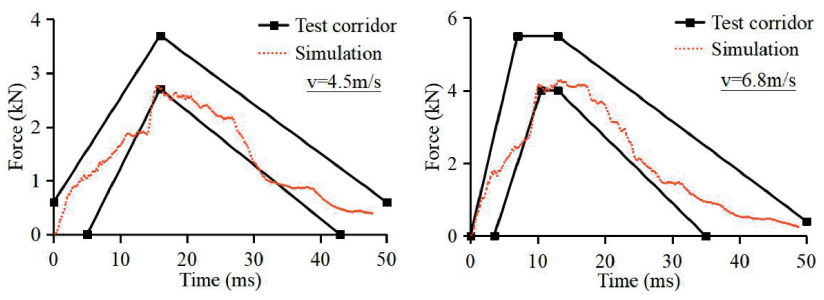

(d)

Fig. 8. Impact force time history curves predicted from THUMS-HS model versus cadaver data:

(a) pelvis, (b) abdomen, (c) thorax and (d) shoulder

In Figure 8, the impact force time history curves predicted from the THUMS-HS model with the corridors from cadaver test data (adapted from Untaroiu et al. [23]) for different body parts are compared. Generally, the predictions are within the corridors excepting some minor errors at the early stage of impact in the abdomen and thorax cases.

\subsection{Validation at the full-body level}

In Figure 9a, the overall kinematics between the THUMS-HS model and a typical cadaver test are compared and in Fig. 9b, the predicted trajectories from the THUMS-HS and THUMS model together with the cadaver test average values are shown [7]. The CORA rating results for each body part of the THUMS-HS model referring to the THUMS model and test average data are also shown in Fig. 9b. Gen- erally, the overall kinematics of the THUMS-HS model matches the cadaver well, though some differences are observed in the lower limbs. The trajectories of the head, T1, T8 and pelvis of the THUMS-HS model are close to those of the THUMS model and cadaver test average data, and the CORA rating results $(>99 \%)$ are all close to full mark.
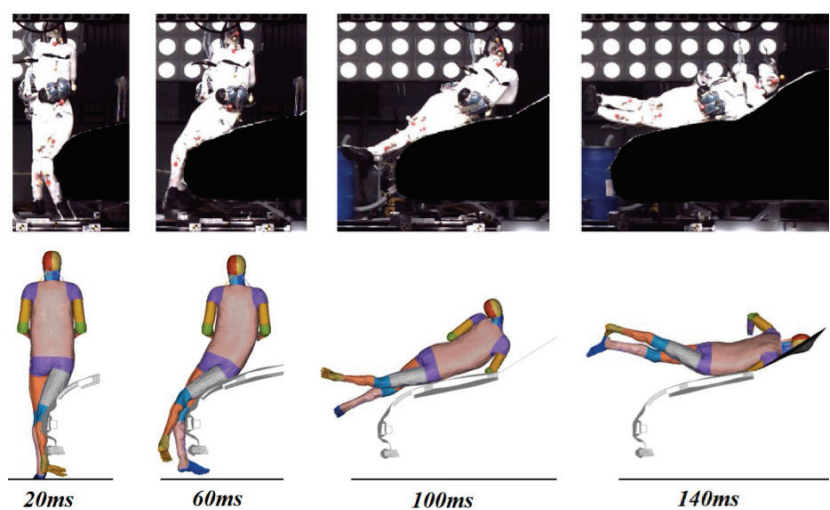

(a)

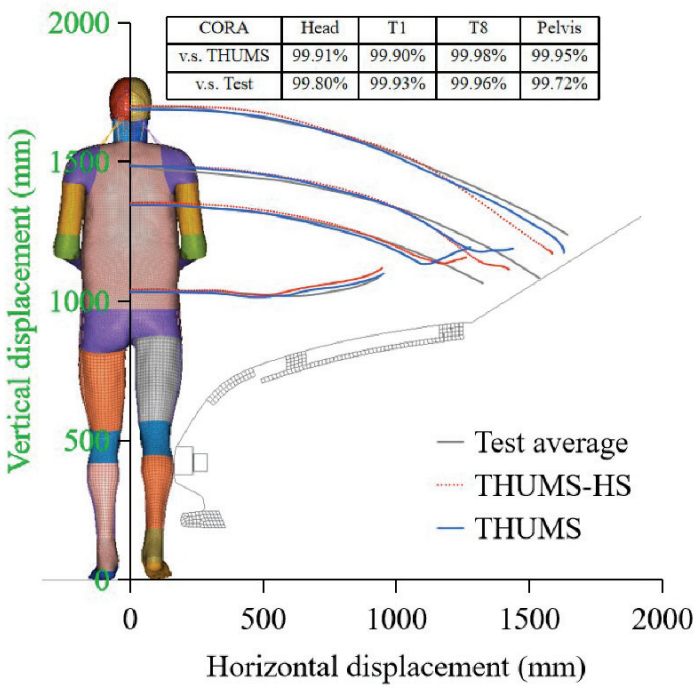

(b)

Fig. 9. Comparison of (a) over kinematics and (b) trajectories of the head, T1, T8 and pelvis between the THUMS-HS model, THUMS model and cadaver test data

\section{Discussion}

\subsection{Capability of the THUMS-HS model}

The current work proposed a novel modeling approach of employing hollow structures to simulate soft tissues in FE human body model and developed the THUMS-HS model using this approach based on 
the THUMS model. The basic concept of using hollow structures to simulate solid soft tissues was evaluated firstly, and the results suggest that this approach could generally predict mechanical response of solid structures when proper material properties are defined (Fig. 2, Table 1). This approach could be employed in other similar human body FE modeling work with the perpose of saving computing time and improving computational stability. The current work is the first attempt to develop a FE pedestrian model using hollow structures to simulate subcutaneous fat and skeletal muscle. The validation results at segment level indicate that the THUMS-HS model has good capability in predicting mechanical response under lateral impact loading for the lower limb, pelvis, abdomen, thorax and shoulder (Figs. 7, 8). The validation results at full-body level also show that the kinematics predicted from the THUMS-HS model matches the cadaver data well (Fig. 9). The above validation method has also been employed in previous studies of developing pedestrian models [10], [23], [33].

The computational efficiency of the THUMS-HS model was compared with the original THUMS model in our preliminary analysis (CPU i7 9700k), where the computing time of the THUMS-HS model (4.5 hours) for a $150 \mathrm{~ms}$ duration vehicle-to-pedestrian impact simulation was only one third of that for the THUMS model (15 hours). This improvement of computational efficiency is due to the reduction of solid element number (760 000 vs. 1400000$)$ and implication of simple material model (plastic material vs. rubber material) in the THUMS-HS model compared with the original THUMS model.

For computational stability, the body tissues that usually suffer excessive deformation under impact loading, e.g., subcutaneous fat and skeletal muscle, were replaced by hollow structures using shell elements in the THUMS-HS model. This can largely reduce the errors from negative volume in solid elements when excessive deformation occurred. To verify the computational stability of the THUMS-HS model, simulations of vehicle-to-pedestrian impact at $70 \mathrm{~km} / \mathrm{h}$ were defined (Fig. 10), where the sedan and SUV front models were extracted from the full scale vehicle models of Toyota Camry 2012 and Toyota RAV4 1997 downloaded from the NCAC website (http://www.ncac.gwu.edu/ vml/models.html). In Figure 11, the predicted overall kinematics of the THUMS-HS model in sedan and SUV crashes at the impact speed of $70 \mathrm{~km} / \mathrm{h}$ were shown, where no solving error was occurred in the simulations, but simulations using the original THUMS model under the same impact loading all terminated

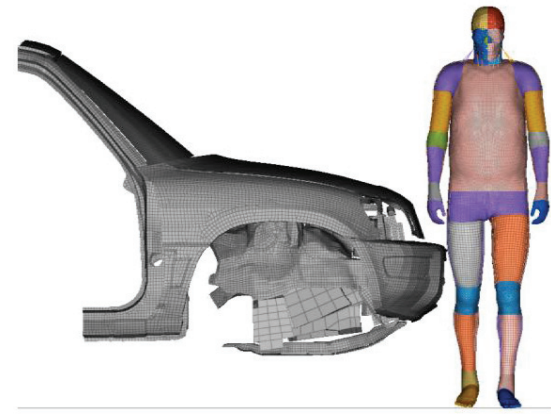

(a)

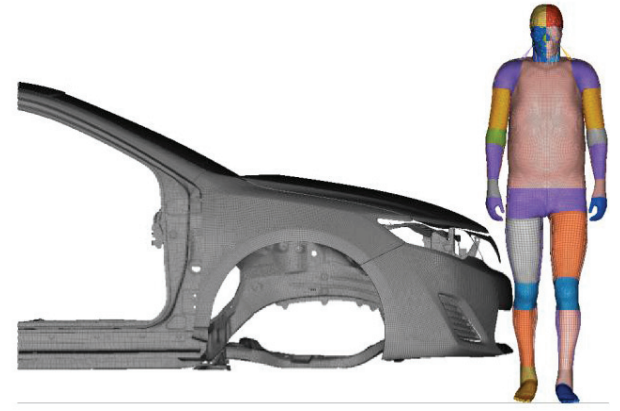

(b)

Fig. 10. Vehicle-to-pedestrian impact simulation setup: SUV crash (a) and sedan crash (b)
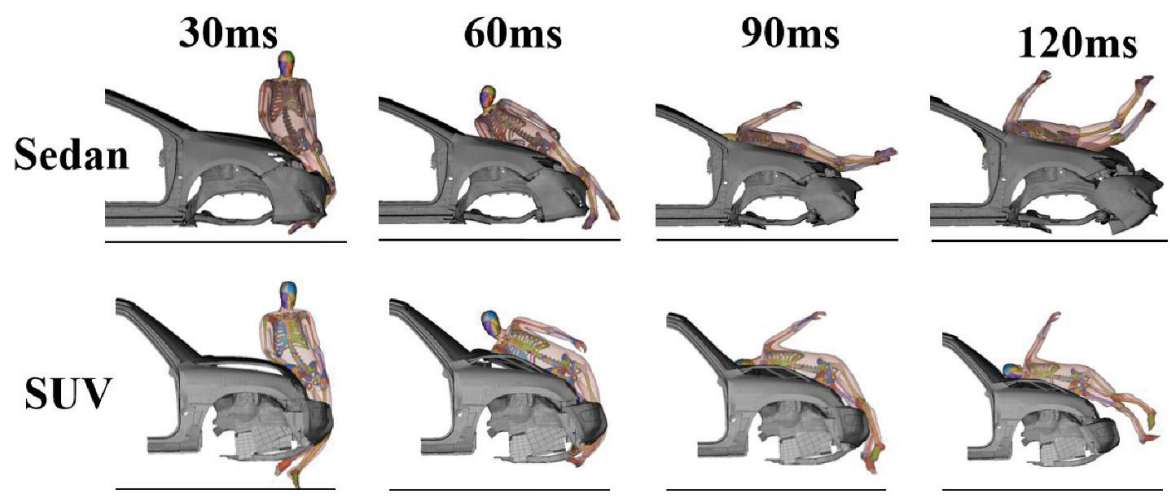

Fig. 11. Kinematics of the THUMS-HS model in sedan and SUV crashes at $70 \mathrm{~km} / \mathrm{h}$ 
abnormality at the early stage of simulation (before $50 \mathrm{~ms}$ ) due to errors of negative volume in solid elements in the lower limb and pelvis.

Previous studies focusing on reduction of element number in detailed human body models have also achieved significant improvement on model computing efficiency [11], [23]. However, the consideration of using hollow structures and simple material model to improve computing efficiency and stability in human body modeling has not been reported before. Besides, the human body modeling method coupling multibody and FE segments can also achieve the goal of high computational efficiency and stability [28], [33]. However, this method needs to replace the coupling parts when studying the injury of different body parts, and the accuracy of the coupling solution from a variety of software is difficult to guarantee. The above findings together with the recognized biofidelity of the THUMS model reported in previous studies [11], [27], [30] imply that the THUMS-HS model could be used as a valid, efficient and robust numerical tool for pedestrian safety analysis in vehicle collisions.

\subsection{Limitations}

It should be noted that the proposed hollow structure together with the specific material properties can generally predict the mechanical response of solid soft tissues under impact loading, but could not be used for modeling soft tissues in detail. The selection of hollow structure, material and properties in the current study is not the unique solution for predicting the mechanical response of human body tissues, users can have their own definition according to loading condition. The compression response in Fig. 2 indicates that the effect of bulk modulus in solid elements could not been predicted by the hollow structure. For model validation, only one impact scenario was considered in validation of the THUMS-HS model at the full body level. However, this is one of the common difficulties in FE human body evaluation given the limited availability of test data [10], [23], [33].

\section{Conclusions}

The current study proposed a novel modeling approach of using hollow structures to simulate the mechanical properties of soft tissues under impact loading, developed a pedestrian FE model (named as
THUMS-HS) employing this modeling approach based on the THUMS pedestrian model, and evaluated the biofidelity and computational efficiency and stability of the THUMS-HS model. The results show that the proposed hollow structures can simulate the mechanical properties of soft tissues under impact loading. The predictions of the THUMS-HS model show good agreements with the cadaver test data at both segment and full body levels. Simulations also prove that the THUMS-HS model has high computational efficiency and stability. The findings indicate that the proposed modeling approach of using hollow structures to simulate the mechanical properties of soft tissues is plausible and the THUMS-HS model could be used as a valid, efficient and robust numerical tool for analysis of pedestrian safety in vehicle collisions.

\section{Acknowledgements}

This study was supported by National Natural Science Foundation of China (Grants No. 51805162 and 31800788), and Scientific Research Fund of Hunan Provincial Education Department (Grant No. 20B233). All simulations using the THUMS and THUMS-HS model in this research were conducted at Shanghai Key Laboratory of Forensic Medicine, Academy of Forensic Science, Ministry of Justic.

\section{References}

[1] C-NCAP. C-NCAP Management Regulation (2021 Version), China New Car Assessment Programme, China Automotive Technology and Research Center, 2020.

[2] Euro-NCAP, Assessment Protocol-Vulnerable Road User Protection, Version 10.0.3, European New Car Assessment Programme, 2020.

[3] Gehre G., Gades H., WernicKe P., Objective rating of signals using test and simulation responses, Proceedings of the 21st International Conference on the Enhanced Safety of Vehicles (ESV), Stuttgart, Germany, 2009.

[4] He Q., Feng J., Zhou H., Tian G., Numerical study on the dynamic behavior of circular honeycomb structure with concentrated filling inclusions defects, J. Mech. Sci. Technol., 2018, 32 (8), 3727-3735.

[5] Ivarsson B., Lessley D., Kerrigan J., Bhalla K., Bose D., Crandall J., Kent R., Dynamic Response Corridors and Injury Thresholds of the Pedestrian Lower Extremities. Proceedings of International Research Council on Biomechanics of Injury (IRCOBI) Conference, Graz, Austria, 2004.

[6] Kerrigan J., Drinkwater D., Kam C., Murphy D., IVARSSON B., Crandall J., PATRIE J., Tolerance of the human leg and thigh in dynamic latero-medial bending, Int. J. Crashworthiness, 2004, 9 (6), 607-623.

[7] Kerrigan J., Murphy D., D Drinkwater., Kam C., Bose D., CRANDAll J., Kinematic corridors for PMHS tested in fullscale pedestrian impact tests. Proceedings of the 19th International Technical Conference of Enhanced Safety of Vehicles (ESV), Washington D.C., USA, 2005. 
[8] Koh S., Cavanaugh J., Mason M., Petersen S., Bolte J., Shoulder injury and response due to lateral glenohumeral joint impact: An analysis of combined data, Stapp Car Crash J., 2005, 49, 291-322.

[9] Li G., Ma H., Guan T., GaO G., Predicting safer vehicle front-end shapes for pedestrian lower limb protection via a $\mathrm{nu}$ merical optimization framework, Int. J. Auto. Tech.-Kor., 2020, 21 (3), 749-756.

[10] Li G., TAN Z., Lv X., REN L., A computationally efficient finite element pedestrian model for head safety: Development and validation, Appl. Bionics Biomech., 2019a, 4930803.

[11] Li G., TAN Z., LV X., REN L., Numerical reconstruction of injuries in a real world minivan-to-pedestrian collision, Acta Bioeng. Biomech., 2019, 21 (2), 21-30.

[12] Li G., Wang F., Otte D., Cai Z., Simms C., Have pedestrian subsystem tests improved passenger car front shape?, Accid. Anal. Prev., 2018, 115, 143-150.

[13] Li G., Wang F., OtTe D., Simms C., Characteristics of pedestrian head injuries observed from real world collision data, Accid. Anal. Prev., 2019c, 129, 362-366.

[14] Luo H., Chen F., Wang X., Dai W., Xiong Y., Yang J., Goog R., A novel two-layer honeycomb sandwich structure absorber with high-performance microwave absorption, Compos. Part A-Appl. S., 2019, 119, 1-7.

[15] LSTC. LS-DYNA keyword user's manual, version 971. Livermore Software Technology Corporation Livermore, United States of America, 2007.

[16] Mo F., Li F., Behr M., Xiao Z., Zhang G., Du X., A lower limb-pelvis finite element model with $3 D$ active muscles, Ann. Biomed. Eng., 2018, 46 (1), 86-96.

[17] Mo F., Zheng Z., Zhang H., Li G., Sun D., In vitro compressive properties of skeletal muscles and inverse finite element analysis: comparison of human versus animals, J. Biomech., 2020, 109, 109916.

[18] NiE J., Li G., YANG J., A study of fatality risk and head $d y$ namic response of cyclist and pedestrian based on passenger car accident data analysis and simulations, Traffic Inj. Prev., 2015, 16 (1), 76-83.

[19] Strandroth J., Sternlund S., Lie A., Tingvall C., Rizzi M., Kullgren A., Ohlin M., FredriKsSON R., Correlation between Euro-NCAP pedestrian test results and injury severity in injury crashes with pedestrians and bicyclists in Sweden, Stapp Car Crash J., 2014, 58, 213-231.

[20] Takhounts E., Craig M., Moorhouse K., McFadden J., HasiJa V., Development of brain injury criteria (BrIC), Stapp Car Crash J., 2013, 57, 243-266.

[21] Toyota Motor Corporation. Documentation: Total Human Model for Safety (THUMS) AM50 Pedestrian/Occupant
Model Academic Version 4.02_20150527, Toyota Center R \& D Labs., Inc, 2015.

[22] Untaroiu C., Shin J., Crandall J., Rikard F., Bostrom O., TAKahashi Y., AkiYama A., Development and validation of pedestrian sedan bucks using finite-element simulations: A numerical investigation of the influence of vehicle automatic braking on the kinematics of the pedestrian involved in vehicle collisions, Int. J. Crashworthiness, 2010, 15 (5), 491-503.

[23] Untaroiu C., Pak W., Meng Y., Schap J., Gayzik F., A finite element model of a midsize male for simulating pedestrian accidents, J. Biomech. Eng.-T. ASME, 2017, 140 (1), 011003.

[24] Untaroiu C., Yue N., Shin J., A finite element model of the lower limb for simulating automotive impacts, Ann. Biomed. Eng., 2012, 41, 1-14.

[25] Vavalle N., Moreno D., Rhyne A., Stitzel J., Gayzik F., Lateral impact validation of a geometrically accurate full body finite element model for blunt injury prediction, Ann. Biomed. Eng., 2013, 41, 497-512.

[26] Viano D., Biomechanical responses and injuries in blunt lateral impact, SAE Technical Paper No. 892432, 1989.

[27] Wang F., Han Y., Wang B., Peng Q., Huang X., Miller K., WitTeK A., Prediction of brain deformations and risk of raumatic brain injury due to closed-head impact: quantitative analysis of the effects of boundary conditions and brain tissue constitutive model, Biomech. Model. Mechan., 2018, 17, 1165-1185.

[28] Wang F., Yu C., Wang B., Li G., Miller K., Wittek A., Prediction of pedestrian brain injury due to vehicle impact using computational biomechanics models: Are head-only models sufficient?, Traffic Inj. Prev., 2020, 21 (1), 102-107.

[29] WHO, Global Status Report on Road Safety, World Health Organization, Geneva, Switzerland, 2015.

[30] Wu T., Kim T., Bollapragada V., Poulard D., Chen H., Evaluation of biofidelity of THUMS pedestrian model under a whole-body impact conditions with a generic sedan buck. Traffic Inj. Prev., 2017, 18, S148-S154.

[31] YANG K., Basic Finite Element Method as Applied to Injury Biomechanics, Elsevier, 2018.

[32] Yang K., Hu J., White N., King A., Chou C., Prasad P., Development of numerical models for injury biomechanics research: A review of 50 years of publications in the Stapp Car Crash conference, Stapp Car Crash J., 2006, 50, 429-490.

[33] Yu C., Wang F., Wang B., Li G., Li F., A computational biomechanics human body model coupling finite element and multibody segments for assessment of head/brain injuries in car-to-pedestrian collisions, Int. J. Env. Res. Pub. He., 2020, 17 (2), 492. 